Case Report

\title{
A Case of Urinary Tract Infection and Severe Sepsis Caused by Kluyvera ascorbata in a 73-Year-Old Female with a Brief Literature Review
}

\author{
Majd Alfreijat \\ Department of Medicine, St. Joseph's Hospital and Medical Center, Phoenix, AZ, USA \\ Correspondence should be addressed to Majd Alfreijat; majd_freijat@yahoo.com
}

Received 24 January 2017; Revised 3 April 2017; Accepted 6 April 2017; Published 3 May 2017

Academic Editor: Alexandre Rodrigues Marra

Copyright (c) 2017 Majd Alfreijat. This is an open access article distributed under the Creative Commons Attribution License, which permits unrestricted use, distribution, and reproduction in any medium, provided the original work is properly cited.

Infections that are caused by Kluyvera bacteria have been previously reported in the medical literature; however, they seem to be less common. Herein, we report a case of urinary tract infection and severe sepsis caused by Kluyvera ascorbata in a 73-year-old female. We also did a brief literature review of infections caused by this organism in adults.

\section{Introduction}

Kluyvera is a Gram-negative bacterium that belongs to the Enterobacteriaceae family. Infections caused by this organism are not very common; however, they have been previously reported in the literature. Herein, we report a case of severe sepsis in a 73-year-old female that was a result of urinary tract infection due to Kluyvera ascorbata.

\section{Case Presentation}

73-year-old female with history of hypertension, and a recent diagnosis of right lower lobe (RLL) lung mass, presented to the emergency department (ED) complaining of progressively worsening dysphagia for few weeks. The patient had difficulty in swallowing both liquids and solids. She reported decreased appetite, weight loss, and multiple episodes of nonbloody vomit. She was scheduled to have an endoscopy as an outpatient on the same day of admission; however, she decided to come to the ED instead, as her symptoms became more severe.

The patient had a remote history of 20 pack-year of cigarettes smoking. Her medications included Aspirin, Iron Pills, Atorvastatin, and Lisinopril. On physical exam, she was hypothermic with a temperature of 35.6 degrees Celsius and tachycardic with a heart rate of 103 . The patient looked cachectic and chronically ill. She also had dry oral mucous membranes. The initial laboratory results showed significant leukocytosis with a white blood cell count of 29.3 thousand/ul and hyponatremia with a sodium of $127 \mathrm{mmol} / \mathrm{L}$. The urine was cloudy in appearance, and it contained leukocytes esterase and more than 50 white blood cells. The chest Xray revealed RLL mass, with no evidence of pulmonary consolidation. The patient was admitted to a telemetry bed and was started on aggressive IV hydration and IV ceftriaxone as a treatment for severe sepsis syndrome. The source of the sepsis was thought to be a urinary tract infection as the urine culture grew Kluyvera ascorbata and Streptococcus agalactiae (group B), and both organisms were sensitive to ceftriaxone. The hospital course was prolonged and complicated. During the first 24 hours, the patient went into atrial fibrillation with rapid ventricular rate and was started on IV amiodarone and heparin drip. She also had an abrupt onset of cold and discolored left leg that required an emergent vascular surgery evaluation. The patient was found to have left femoral artery occlusion and underwent left iliac artery stenting and left femoral endarterectomy.

Furthermore, she had a CT-guided biopsy of the RLL mass and the pathology was consistent with primary adenocarcinoma. The patient was intubated for the surgical procedure and the biopsy. Few days later, she was briefly extubated; however, she continued to have significant hypoxia and hypotension. There was also a concern for aspiration pneumonia. Palliative care team was involved and after an 
extensive discussion with family, the decision was taken to change the code status to DNR. Patient expired after 2 weeks of hospitalization. The endoscopy was never done as the patient was never in a stable condition throughout her hospital stay.

\section{Discussion}

Kluyvera is a group of Gram-negative rods bacteria and a member of the family Enterobacteriaceae [1]. It was named after Albert Jan Kluyver, a prominent Dutch microbiologist who was the first to propose its existence in 1936 [2]. The organism was isolated from soil and sewage by Asai et al. in 1956 [3]; however, it was not until 1981 that its molecular characteristics were defined by Farmer et al. [4]. Kluyvera genus has four species: Kluyvera ascorbata, Kluyvera cryocrescens, and Kluyvera georgiana that were all found in humans and Kluyvera cochleae that was isolated from snails and slugs [5].

Between 1980 and 2005 there have been 41 cases of clinically significant infections in humans caused by Kluyvera: 21 of them were due to $K$. ascorbata, 8 due to $K$. cryocrescens, and 12 were due to an unspecified Kluyvera [6].

In 2001 Sarria et al. published the largest retrospective analysis about the clinical manifestations of infections caused by Kluyvera and that included gastroenteritis (diarrhea and fever), acute pancreatitis, bacteremia, wound infection, urinary tract infection (UTI), pyelonephritis, acute cholecystitis, peritonitis, mediastinitis, infected urethrorectal fistula, and soft tissue infection [7]. Since 2005, and to the best of our knowledge, there have been only 6 other cases of Kluyvera ascorbata infection in adults manifested as UTI, pyelonephritis, sepsis, liver abscess, and bacteremia [8-12]. Interestingly, in one of these cases the bacteria were multidrug resistant [11].

\section{Conclusion}

It is essential to report common conditions like UTIs that are caused by less common microbes like Kluyvera ascorbata. This could help in raising awareness of this organism in the medical society.

\section{Consent}

An informed patient consent was obtained.

\section{Conflicts of Interest}

The author declares that there are no conflicts of interest.

\section{References}

[1] T. Asai, H. lizuka, and K. Kômagata, "The flagellation of genus Kluyvera," The Journal of General and Applied Microbiology, vol. 8, pp. 187-191, 1962.

[2] A. J. Kluyver and C. B. van Niel, "Prospects for a natural system of classification of bacteria. Zentralbl Bakteriol Parasitenkd Infektionskr Hyg Abt II," vol. 94, pp. 369-403, 1936.
[3] A. Toshinobu, S. Okumura, and T. Tsunoda, "On a new genus," Proceedings of the Japan Academy, vol. 32, no. 7, pp. 488-493.

[4] J. J. Farmer et al., "Kluyvera, a new (redefined) genus in the family Enterobacteriaceae: identification of Kluyvera ascorbata sp. nov. and Kluyvera cryocrescens sp. nov. in clinical specimens," Journal of Clinical Microbiology, vol. 13, no. 5, pp. 919-933, 1981.

[5] H. E. Müller, D. J. Brenner, G. R. Fanning, P. A. D. Grimont, and P. Kampfer, "Emended description of Buttiauxella agrestis with recognition of six new species of Buttiauxella and two new species of Kluyvera: Buttiauxella ferragutiae sp. nov., Buttiauxella gaviniae sp. nov., Buttiauxella brennerae sp. nov., Buttiauxella izardii sp. nov., Buttiauxella noackiae sp. nov., Buttiauxella warmboldiae sp. nov., Kluyvera cochleae sp. nov., and Kluyvera georgiana sp. nov.," International Journal of Systematic Bacteriology, vol. 46, no. 1, pp. 50-63, 1996.

[6] J. E. Carter and T. N. Evans, "Clinically significant kluyvera infections: a report of seven cases," American Journal of Clinical Pathology, vol. 123, no. 3, pp. 334-338, 2005.

[7] J. C. Sarria, A. M. Vidal, and R. C. Kimbrough, "Infections caused by Kluyvera species in humans," Clinical Infectious Diseases, vol. 33, no. 7, pp. e69-e74, 2001.

[8] D. Torre, E. Crespi, M. Bernasconi, and P. Rapazzini, "Urinary tract infection caused by Kluyvera ascorbata in an immunocompromised patient: case report and review," Scandinavian Journal of Infectious Diseases, vol. 37, no. 5, pp. 375-378, 2005.

[9] R. Cheruvattath, V. Balan, R. Stewart, R. L. Heilman, D. C. Mulligan, and S. Kusne, "Kluyvera co-infection in two solid organ transplant recipients: an emerging pathogen or a colonizer bystander?" Transplant Infectious Disease, vol. 9, no. 1, pp. 83-86, 2007.

[10] E. R. Carrillo, P. C . Peña, B. J. Muciño, C. J. R. Carrillo, and C. L. D. Carrillo, "Severe sepsis, septic shock and secondary multiple organ dysfunction in infection by Kluyvera ascorbata," Gaceta Medica de Mexico, vol. 147, no. 4, pp. 355-360, 2010.

[11] S. Moonah, K. Deonarine, and C. Freeman, "Multidrug resistant Kluyvera ascorbata septicemia in an adult patient: a case report," Journal of Medical Case Reports, vol. 4, article 197, 2010.

[12] G. López-Larramona, E. Gómez-de-Oña, M. M. MaestreMuñiz, A. M. Ruiz-Chicote, E. Galán-Dorado, and L. GonzálezDelgado, "Kluyvera ascorbata bacteremia in an adult patient," Revista española de quimioterapia: publicación oficial de la Sociedad Española de Quimioterapia, vol. 26, no. 3, pp. 226-227, 2013. 


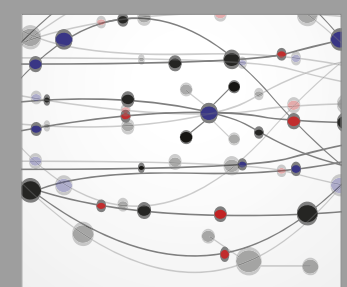

The Scientific World Journal
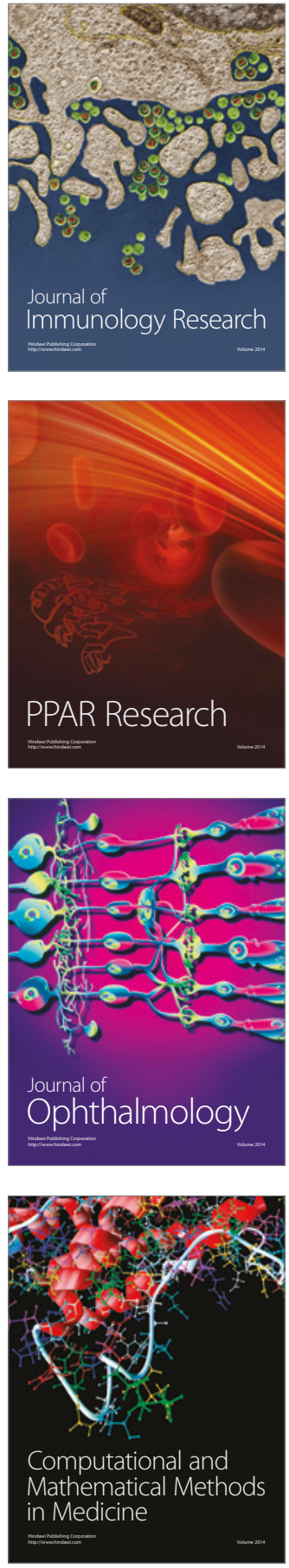

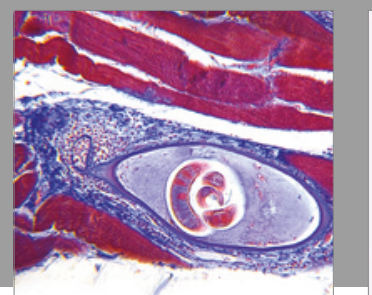

Gastroenterology Research and Practice
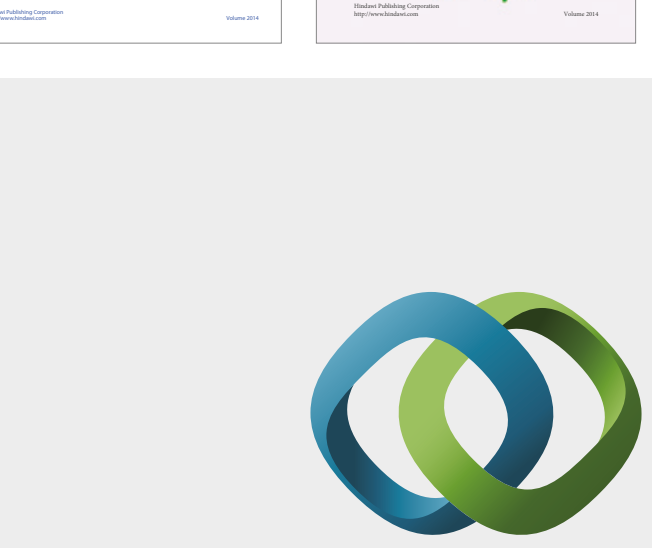

\section{Hindawi}

Submit your manuscripts at

https://www.hindawi.com
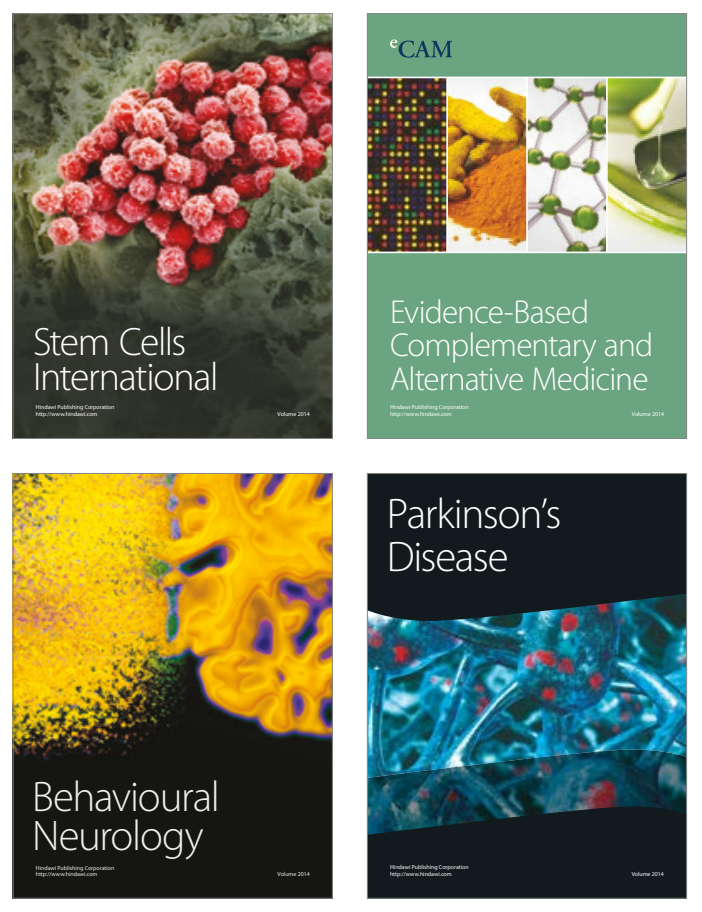
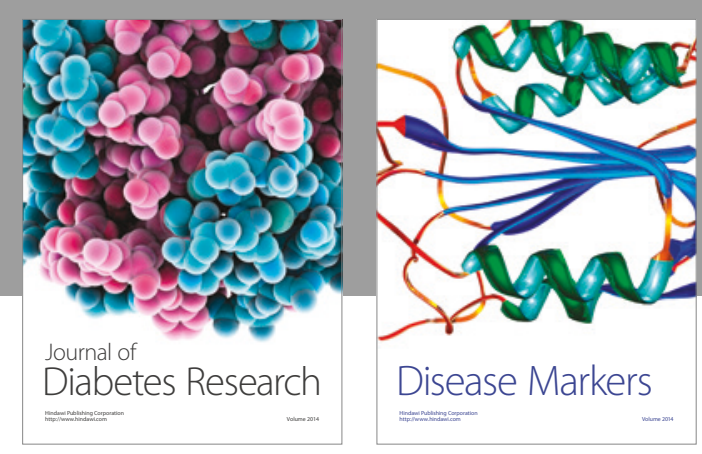

Disease Markers
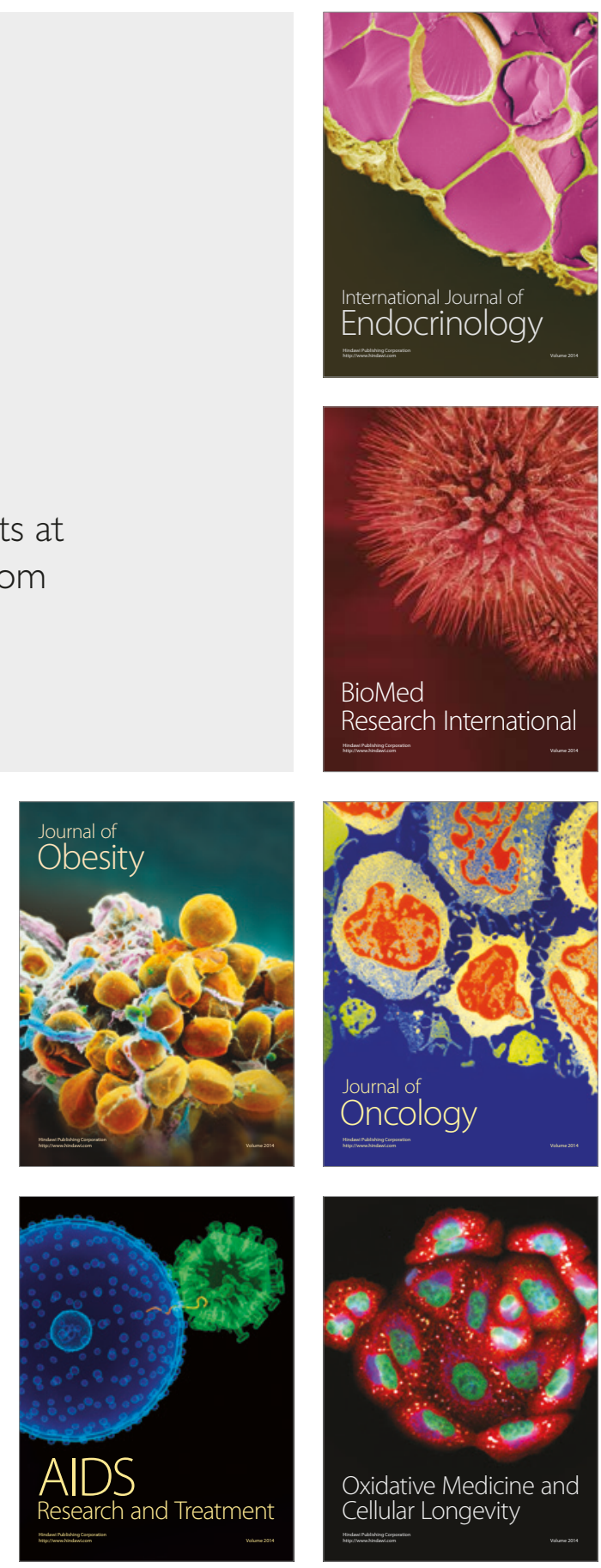